\title{
Designing Caspase-3 Sensors for Imaging of Apoptosis in Living Cells
}

\author{
Ning Chen ${ }^{[a]}$, Yun Huang ${ }^{[a]}$, Lily Yang ${ }^{[b]}$, Rihe Liu[c], and Jenny J. Yang ${ }^{[a]}$ \\ Jenny J. Yang: chejjy@langate.gsu.edu \\ [a]Department of Chemistry, Center for Biotechnology and Drug Design, Georgia State University, \\ Atlanta, GA 30303 (USA), Fax: (+1)404-413-5551 \\ [b]Department of Surgery and Winship Cancer Institute, School of Medicine, Emory University, \\ Atlanta, GA 30322 (USA) \\ [c]School of Pharmacy, The University of North Carolina at Chapel Hill, NC 27599 (USA)
}

\section{Keywords}

apoptosis; caspase-3; enhanced green fluorescent proteins; protease sensors; ratiometric measurements

Apoptosis is an essential physiological process of tissue development and homeostasis and is associated with pathogenesis of human diseases, such as neurodegenerative diseases and cancers. ${ }^{[1,2]}$ The caspase-cascade system plays a vital role in the induction, signal transduction, and amplification of intracellular apoptotic signals. ${ }^{[3-5]}$ One of the challenges in monitoring apoptosis pathways includes the serial involvement of multiple caspases, complicated activation or inhibition mechanisms, and the heterogeneous cell population at a given time. Therefore, the mechanism of caspase activation during apoptosis is not fully understood and investigations have been hampered by a lack of real-time sensors with high enzymatic specificity to quantitatively detect caspase activity in live cells or in vivo. ${ }^{[6,7]}$

Currently, the majority of methods for detecting caspase activity include Western blot to detect the cleaved products, or synthetic substrates conjugated to a chromogenic dye to monitor caspase activity in cell lysates during apoptosis. ${ }^{[6-8]}$ Some newly developed peptide probes with cell permeability allow one time snap-shots of caspase activity. ${ }^{[9]}$ A DNA dye, DEVD-NucView 488 substrate with a DEVD cleavage linker, allows an indirect report of the activation of caspase- 3 after diffusion to nuclei in living cells. ${ }^{[10]}$ To achieve real-time ratiometric signal changes during caspase activation, progress has been made to develop FRET-based (FRET, fluorescence resonance energy transfer) probes by connecting two fluorescent protein (FP) pairs with a caspase cleavage linker. ${ }^{[11,12]}$

Here, we describe a sensitive and specific caspase- 3 sensor, based on a single enhanced green fluorescent protein (EGFP) to avoid the cross-talk from the overlap of cyan fluorescent protein (CFP) excitation or yellow fluorescent protein (YFP) emission spectra as observed in most FRET probes. ${ }^{[11]}$ A ratiometric protease sensor was designed by grafting a caspase-3-specific cleavage linker at a sensitive loop location (Glu172) relative to the chromophore, taking advantage of the EGFP's high resistance to proteases (Figure 1a). ${ }^{[13]}$

(C) 2009 Wiley-VCH Verlag GmbH\&Co. KGaA, Weinheim

Correspondence to: Jenny J. Yang, chejjy@langate.gsu.edu.

Supporting information for this article is available on the WWW under http://dx.doi.org/10.1002/chem.200901439. 
Previously we have shown that calcium and trypsin sensors can be created by grafting a calcium-binding loop or trypsin cleavage site at Glu172 of EGFP, respectively. ${ }^{[14,15]}$ Addition of a protease-specific cleavage linker at Glu172 loop region of EGFP results in formation of dual chromophore states with absorption at both 397 and $490 \mathrm{~nm} .{ }^{[16]}$ Such an equilibrium between the protonated and deprotonated chromophore forms was proposed to originate from a complicated hydrogen-bond network allowing for proton transfer between the chromophore and its neighboring side chains. The predominant protonated form of the chromophore with its maximum absorbance at $397 \mathrm{~nm}$ is governed by the carboxylation of Glu222 through electrostatic repulsion and hydrogen bonding between a bound water molecule and Ser205. On the other hand, the maximum absorbance at $490 \mathrm{~nm}$ of the deprotonated form of the chromophore is likely to be due to the proton relay between Glu222 and Ser205. The side chains of Asp117, Thr118, Glu172, and Asp190 may also be involved in the alteration of different conformations of the chromophore under the observation of electron density of crystal structure. Our observation of the switch of dual chromophore forms upon the insertion of a cleavage linker at position 172 is probably a result of the alteration of the ionization state of the chromophore. ${ }^{[14,15]}$

Three EGFP-based caspase-3 sensor variants were genetically generated (Figure 1b). To obtain optimal signal change and kinetic properties, two helical sequences were used to extend the caspase- 3 cleavage linker and to improve enzymatic accessibility in solution based on our previous knowledge gained in developing calcium and trypsin sensors. ${ }^{[14,15]}$ The insertion of sequence with helical properties results in a significantly greater signal change, possibly due to its greater solvent accessibility for calcium binding and trypsin cleavage. The comparison between dual cleavage sequence (DEVD) and a single sequence in caspase- 3 sensors was also conducted. No caspase-3 cleavage was observed with addition of only a single DEVD or DEVD without using a helical sequence. Thus, two cleavage sequences (DEVD) grafted onto EGFP were used to obtain a sensor, designated EGFP-C3A, for achieving rapid cleavage. Moreover, to achieve enzymatic specificity, a recognition sequence (VDEVDG) with preferable residues from $\mathrm{P}_{5}$ to $\mathrm{P}_{1}{ }^{\prime}$ was inserted into EGFP to create another sensor denoted as EGFP-C3B ${ }^{[17]}$ Finally, a negative control, EGFP-C3N, was created by grafting a loop lacking a caspase- 3 cleavage site.

Previous investigations showed that protease sensors designed by EGFP through the grafting of a cleavable linker for specific proteases can be cleaved at the designed location without the release of other small fragments under physiological conditions; these cleavage sites were identified by using protein sequence analysis, MALDI-MS and SDS-PAGE. Moreover, the cleaved fragments exhibited strong fluorescence complementation in living cells. ${ }^{[18]}$ Similarly, both purified EGFP-C3A and EGFP-C3B were specifically cleaved by caspase-3 into two major fragments in caspase-3 reaction buffer (20 mм HEPES, $100 \mathrm{~m}_{\mathrm{M} \mathrm{NaCl}}, 1 \mathrm{~m}_{\mathrm{M}}$ EDTA, 10 mм DTT, 10\% sucrose, pH 7.5), as revealed by SDS-PAGE (Figure 1c). Mass spectrometry analysis reveals that the molecular mass of the cleavage fragments is consistent with cleavage at the desired caspase-3 cleavage location (Figure 1d). In contrast, wild type EGFP (EGFP-wt) and EGFP-C3N did not exhibit similar cleavage upon overnight incubation with caspase-3. Moreover, our caspase- 3 sensors can not be cleaved by caspase-6, caspase-7, caspase-8, and caspase-9 (Figure 1c). Such strong enzymatic specificity is very important for its further application in monitoring caspase-3 action in live systems and understanding the mechanisms that control the apoptosis cascade.

Cancer cell lines, HeLa or MIA PaCa-2 cells transfected with EGFP-C3A, EGFP-wt or EGFP-C3N exhibit strong fluorescence at $510 \mathrm{~nm}$ when excited at either 398 or $488 \mathrm{~nm}$ following 18 to $48 \mathrm{~h}$ of expression (Figure 2a,b). Upon caspase-3 activation by $1 \mu_{\mathrm{M}}$ staurosporine (STS), the fluorescence emission signal of EGFP-C3A at $510 \mathrm{~nm}$ exhibited a significant increase with excitation of $398 \mathrm{~nm}$ and a concurrent decrease with excitation of 
$488 \mathrm{~nm}$ (Figure $2 \mathrm{a}-\mathrm{c})$. Such ratiometric fluorescence change $\left(F_{398 \mathrm{~nm}} / F_{488 \mathrm{~nm}}\right)$ has a dynamic range up to 3.2 in HeLa cells and 7.5 in MIA PaCa-2 cells (Figure 2d), which is consistent with observations that lower caspase- 3 activation was detected in cell lysates of HeLa cells than in MIA PaCa-2 cells using a fluorogenic caspase-3 substrate kit, Ac-DEVD-AFC (Supporting Information Figure S1a) or Western blot through caspase-3 antibody (Figure 2e). Moreover, this result is also in agreement with qualitative detection of caspase- 3 activity by immunofluorescence methods ${ }^{[19]}$ and the detection with DEVD-NucView 488 caspase-3 substrate (Supporting Information Figure S1b). In contrast, at $510 \mathrm{~nm}$, no significant fluorescence signal change of EGFP-wt or EGFP-C3N, which lacks cleavage sites, was observed with excitation of 398 and $488 \mathrm{~nm}$ (Figure 2d). Similar results can also be obtained by using fluorescence spectroscopy to monitor emission spectra changes of living mammalian cells expressing the caspase-3 sensor gene in the coverslip following STS induction (Supporting Information Figure S2). The cells transfected with EGFP-C3A following incubation with the caspase-3 inhibitor, Ac-DEVD-CHO, exhibited no ratiometric signal change.

Our developed sensors exhibit a capability of monitoring the early stage of an apoptotic event. MIA PaCa- 2 cells exhibit a half time of $16.8 \pm 3.5$ min for caspase- 3 activation, which is shorter than HeLa cells (30.2 $\pm 5.4 \mathrm{~min}$ ) as detected by EGFP-C3A (Figure 2d). In addition, the half time of caspase- 3 activation is $16.3 \pm 5.9$ min as detected by EGFP-C3B, which is shorter than $48.5 \pm 11.2$ min with commercially available DEVD-NucView 488 caspase- 3 substrate detection under identical inducer concentration in HeLa cells (Figure 2 f). This is due to the direct monitoring of caspase- 3 action in the cytosol by our sensor, rather than detection of caspase-3 after its migration from cytosol to nuclei to accomplish DNA dye cleavage and cooperative DNA binding in an on/off mode. In addition, caspase-3 activation was observed following the migration from the cytoplasm to the nucleus after STS induction based on the fluorescence signal change (Supporting Information: movie S1), which is in good agreement with previous reports of caspase- 3 activation pattern in living cells. ${ }^{[5,20]}$

Table 1 summarizes kinetic parameters, turnover number $\left(k_{\text {cat }}\right)$, Michaelis constants $\left(K_{\mathrm{M}}\right)$, and substrate specificity constants $\left(k_{\text {cat }} / K_{\mathrm{M}}\right)$ of our caspase- 3 sensors and commercially available caspase- 3 substrates determined in caspase-3 reaction buffer. EGFP-C3B exhibited a smaller $K_{\mathrm{M}}$ and a higher $k_{\mathrm{cat}} / K_{\mathrm{M}}$ than commercial caspase-3 substrates, Bz-DEVD-pNA and Z-DEVD-AMC from previous reports. ${ }^{[21]}$ Overall, EGFP-C3B with optimal cleavage residues from $\mathrm{P}_{5}$ to $\mathrm{P}_{1}{ }^{\prime}$ exhibited a larger dynamic range as well as better kinetic properties as indicated by the $k_{\mathrm{cat}} / K_{\mathrm{M}}$ value. As shown in Table 1 , the $k_{\mathrm{cat}} / K_{\mathrm{M}}$ value of EGFP-C3B $\left(59600 \pm 9300 \mathrm{M}^{-1} \mathrm{~s}^{-1}\right)$ is fivefold larger than that of EGFP-C3A $\left(12500 \pm 150_{\mathrm{M}}^{-1} \mathrm{~s}^{-1}\right)$.

In summary, we have developed ratiometric caspase- 3 sensors by grafting cleavage linkers at a location sensitive to chromophore optical signal ratiometric change of a single EGFP. The designed caspase- 3 sensor can be used to detect caspase- 3 activation or inhibition in living cells with a large dynamic range and high enzymatic specificity due to high potential accessibility for caspase- 3 at a lower basal caspase-3 activity or caspase-3 activation in living cells at the early stage of apoptosis, which can eliminate interferences from background, expression level, and problems of orientation change or translocation. In addition, this strategy can also be specifically targeted to desired cellular compartments with the aid of specific signal peptides for real-time monitoring of caspase- 3 activation or inhibition, which will be valuable for investigations of physiological mechanisms or pathological processes of diseases related to caspase- 3 activity at early stage of apoptosis in living cells or in vivo. Because the newly developed sensors are ratiometric and not FRETbased, they are applicable over a slightly wider range to avoid cross-talk from the overlap of CFP excitation or YFP emission spectra, which occurs in most FRET probes. Furthermore, 
this novel strategy for the design of caspase- 3 sensors can also be extended to the development of other specific caspase sensors such as caspase- 8 , caspase- 6 and caspase- 9 sensors, facilitating drug development and protease inhibitor screening.

\section{Experimental Section}

The detailed information related to design, expression, purification, kinetic studies of caspase sensors, real-time detection of caspase-3 activation using designed caspase-3 sensor in living cells and confirmation for caspase-3 in cells is given in the Supporting Information.

\section{Supplementary Material}

Refer to Web version on PubMed Central for supplementary material.

\section{Acknowledgments}

We would like to thank Dan Adams and Michael Kirberger for their critical review of this manuscript, Jin Zou, Shenghui Xue, Yiming Ye, and Shen Tang for helpful discussions; and other members of Dr. Yang's research group for their helpful discussions and suggestions. This work is supported in part by the following sponsors: GM-70555 to J.J.Y.; GSU Molecular Basis of Disease Predoctoral Fellowships to N.C.

\section{References}

1. Bredesen DE. Curr Mol Med. 2008; 8:173-186. [PubMed: 18473818]

2. Jiang X, Wang X. Annu Rev Biochem. 2004; 73:87-106. [PubMed: 15189137]

3. Degterev A, Boyce M, Yuan J. Oncogene. 2003; 22:8543-8567. [PubMed: 14634618]

4. Salvesen GS, Riedl SJ. Adv Exp Med Biol. 2008; 615:13-23. [PubMed: 18437889]

5. Sundararajan R, Chen G, Mukherjee C, White E. Oncogene. 2005; 24:4908-4920. [PubMed: 15824730]

6. Gurtu V, Kain SR, Zhang G. Anal Biochem. 1997; 251:98-102. [PubMed: 9300088]

7. Kohler C, Orrenius S, Zhivotovsky B. J Immunol Methods. 2002; 265:97-110. [PubMed: 12072181]

8. Thornberry NA, Rano TA, Peterson EP, Rasper DM, Timkey T, Garcia-Calvo M, Houtzager VM, Nordstrom PA, Roy S, Vaillancourt JP, Chapman KT, Nicholson DW. J Biol Chem. 1997; 272:17907-17911. [PubMed: 9218414]

9. Wang ZQ, Liao J, Diwu Z. Bioorg Med Chem Lett. 2005; 15:2335-2338. [PubMed: 15837320]

10. Cen H, Mao F, Aronchik I, Fuentes RJ, Firestone GL. FASEB J. 2008; 22:2243-2252. [PubMed: 18263700]

11. Ai HW, Hazelwood KL, Davidson MW, Campbell RE. Nat Methods. 2008; 5:401-403. [PubMed: 18425137]

12. Nagai T, Miyawaki A. Biochem Biophys Res Commun. 2004; 319:72-77. [PubMed: 15158444]

13. Chiang CF, Okou DT, Griffin TB, Verret CR, Williams MN. Arch Biochem Biophys. 2001; 394:229-235. [PubMed: 11594737]

14. Chen N, Zou J, Wang S, Ye Y, Huang Y, Gadda G, Yang JJ. Biochemistry. 2009; 48:3519-3526. [PubMed: 19271729]

15. Zou J, Hofer AM, Lurtz MM, Gadda G, Ellis AL, Chen N, Huang Y, Holder A, Ye Y, Louis CF, Welshhans K, Rehder V, Yang JJ. Biochemistry. 2007; 46:12275-12288. [PubMed: 17924653]

16. Brejc K, Sixma TK, Kitts PA, Kain SR, Tsien RY, Ormo M, Remington SJ. Proc Natl Acad Sci USA. 1997; 94:2306-2311. [PubMed: 9122190]

17. Nicholson DW. Cell Death Differ. 1999; 6:1028-1042. [PubMed: 10578171]

18. Chen N, Ye Y, Zou J, Li S, Wang S, Martin A, Wohlhueter R, Yang JJ. J Biotechnol. 2009; 142:205-213. [PubMed: 19500621]

19. Yang L, Cao Z, Yan H, Wood WC. Cancer Res. 2003; 63:6815-6824. [PubMed: 14583479] 
20. Ramuz O, Isnardon D, Devilard E, Charafe-Jauffret E, Hassoun J, Birg F, Xerri L. Int J Exp Pathol. 2003; 84:75-81. [PubMed: 12801281]

21. Fang B, Boross PI, Tozser J, Weber IT. J Mol Biol. 2006; 360:654-666. [PubMed: 16781734] 

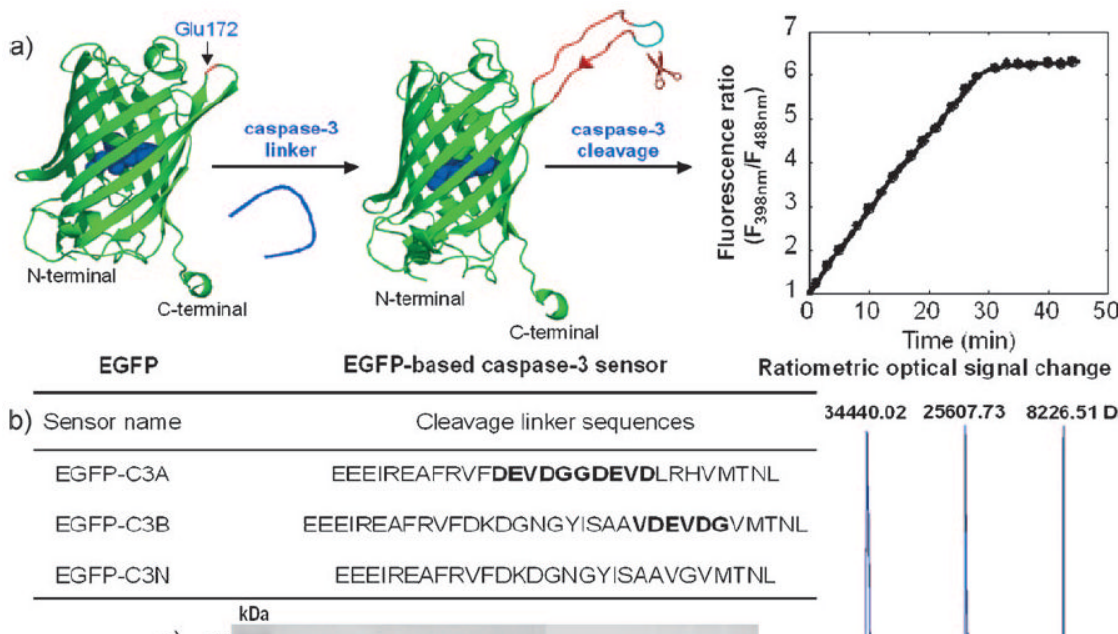

c)
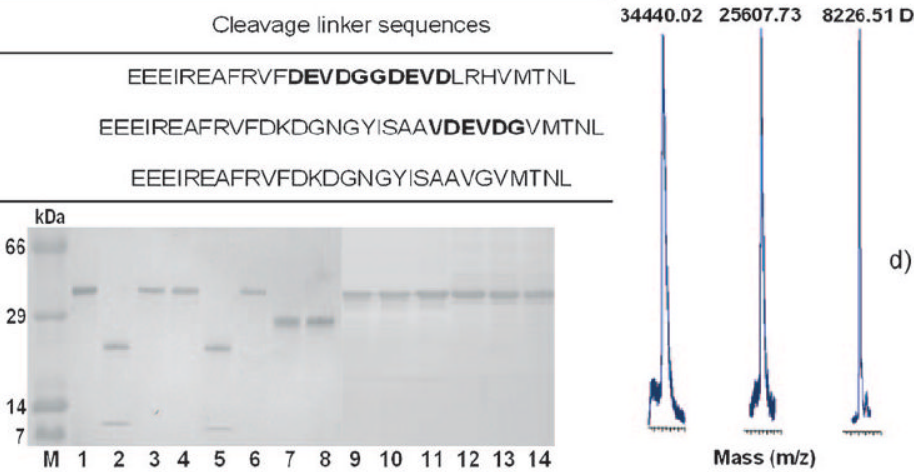

Figure 1.

Design of EGFP-based caspase-3 sensors. a) The model and b) cleavage linkers of ratiometric EGFP-based caspase-3 sensors via grafting approach are demonstrated. c) The specificity of caspase-3 sensors was detected in SDS-PAGE. d) The cleavage sites were identified by MALDI-MS. Lanes M, 1, 2, 3, 4, 5, 6, 7, 8, 9, 10, 11, 12, 13 and 14 are: protein marker, EGFP-C3A, EGFP-C3A with caspase-3 digestion, EGFP-C3A with caspase-8 digestion, EGFP-C3B, EGFP-C3B with caspase-3 digestion, EGFP-C3B with caspase-8 digestion, EGFP-wt, EGFP-wt with caspase-3 digestion, EGFP-C3A with caspase-6 digestion, EGFP-C3A with caspase-9 digestion, EGFP-C3A with caspase-7 digestion, EGFP-C3B with caspase-6 digestion, EGFP-C3B with caspase-9 digestion and EGFP-C3B with caspase-7 digestion, respectively. The molecular masses of intact EGFP-C3A, cleaved major large fragment and major small fragment are 34440.02, 25607.73 and $8226.51 \mathrm{Da}$, respectively. 

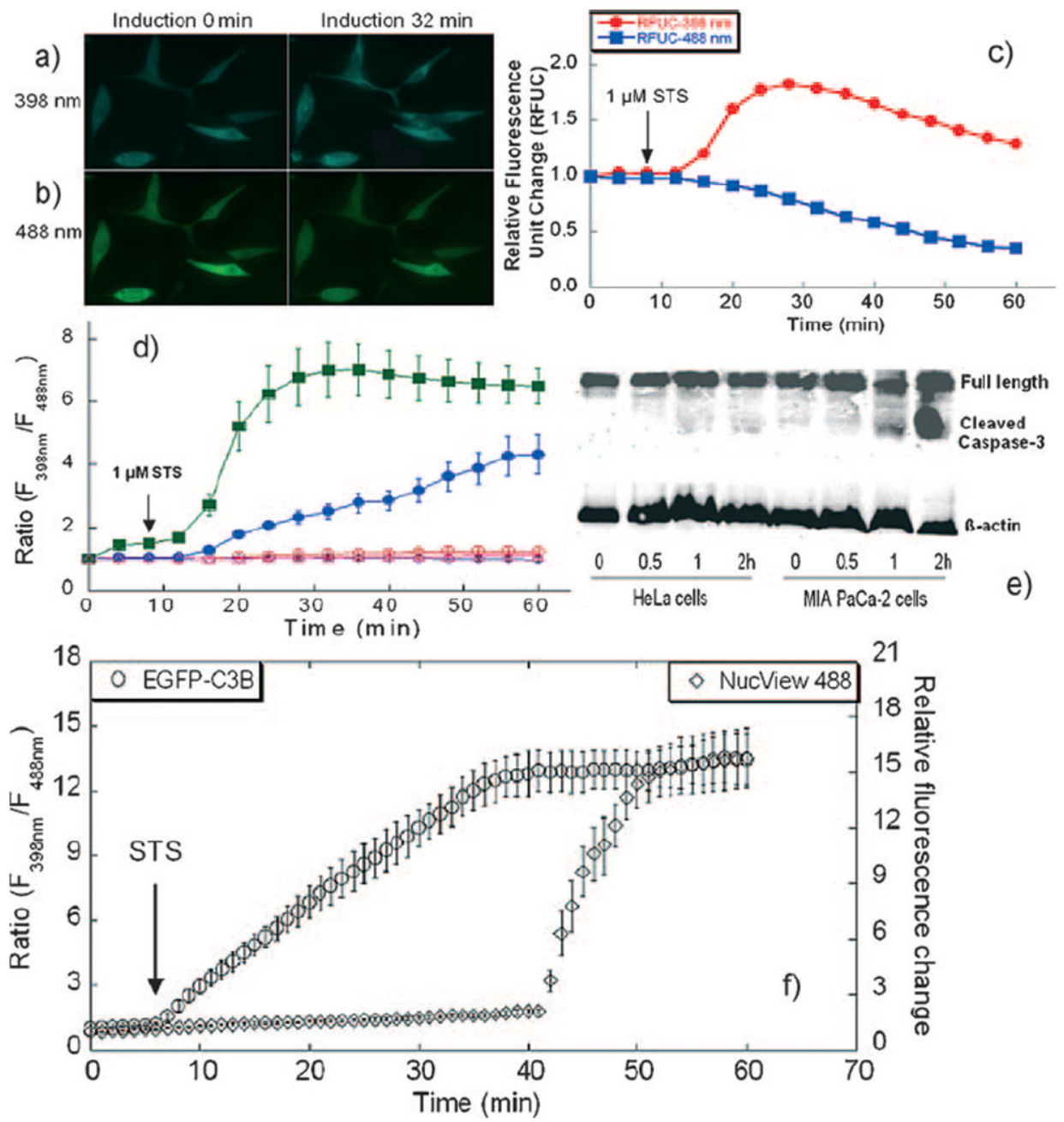

Figure 2.

Live-cell imaging and Western blot analysis of caspase-3 activity. HeLa cells transiently transfected with EGFP-C3A were examined by fluorescence microscope with excitations of a) 398 and b) $488 \mathrm{~nm}$. A significant fluorescence increase was observed between 0-32 min post-induction of $1 \mu_{\mathrm{M}} \mathrm{STS}$ at $398 \mathrm{~nm}$, with a concurrent fluorescence decrease from 0-32 min post-induction of $1 \mu_{\mathrm{M}} \mathrm{STS}$ at $488 \mathrm{~nm}$. c) The relative fluorescence changes at excitations of $398(\bullet)$ and $488 \mathrm{~nm}(\boldsymbol{\square})$ were shown as a ratiometric mode upon addition of 1 $\mu_{M}$ STS. d) Fluorescence ratio change among EGFP-wt (O), EGFP-C3A with Ac-DEVDCHO inhibition $(\diamond)$, EGFP-C3N without cleavage sites $(\Delta)$, EGFP-C3A in HeLa cells $(\bullet)$ and in MIA PaCa-2 cells ( $\mathbf{\square})$ following STS induction reveals a significantly shorter half time for caspase- 3 activation in MIA PaCa- 2 cells. e) Caspase- 3 activation in HeLa and MIA PaCa- 2 cells exhibited a faster caspase- 3 activation and a larger proportion of activated caspase- 3 in MIA PaCa- 2 cells following $1 \mu_{\mathrm{M}}$ STS induction by means of a Western blot analysis. f) EGFP-C3B with an optimal cleavage linker was observed to have a shorter half time (16.3 $\pm 5.9 \mathrm{~min})$ than DEVD-NucView 488 caspase-3 substrate $(48.5 \pm 11.2 \mathrm{~min})$ in HeLa cells following $1 \mu_{\mathrm{M}}$ STS stimulation. 
Table 1

Catalytic kinetic parameters of caspase-3 sensors.

\begin{tabular}{llll}
\hline Sensor & $\boldsymbol{k}_{\text {cat }}\left[\mathrm{s}^{-1}\right]$ & $\boldsymbol{K}_{\mathrm{M}}[\boldsymbol{\mu M}]$ & $\boldsymbol{k}_{\text {cat }} / \boldsymbol{K}_{\mathbf{M}}\left[\mathrm{M}^{-1} \mathbf{s}^{-1}\right]$ \\
\hline EGFP-C3A & $0.13 \pm 0.01$ & $10.03 \pm 1.19$ & $12500 \pm 150$ \\
EGFP-C3B & $0.39 \pm 0.03$ & $6.55 \pm 1.53$ & $59600 \pm 9300$ \\
Bz-DEVD-pNA & $0.73 \pm 0.02$ & $26.91 \pm 1.20$ & $2730 \pm 120$ \\
\hline
\end{tabular}

